\title{
Use of Lorcaserin, A 5HT2C Agonist, In the Management of Olanzapine- Induced Weight Gain
}

\section{Charles Nguyen ${ }^{1}$, Amanda Suzuki $^{2}$ and Kevin Bera ${ }^{3 *}$}

${ }^{1}$ UC Irvine Medical Center, USA

${ }^{2}$ Department of Psychiatry at UC Irvine, USA

${ }^{3}$ University of Southern California, USA

\begin{abstract}
Atypical antipsychotics are associated with weight gain, which compromise medication adherence and pose longterm health complications. These are two cases in which lorcaserin, a 5HT2C agonist, has been used to counteract olanzapine-induced weight gain in conjunction with a low carbohydrate diet. In Case 1, patient lost a significant amount of weight and reported decreased food cravings. In Case 2, patient showed initial response but was unable to maintain diet. Patient had significant weight gain and reported increased cravings after discontinuing lorcaserin. These cases demonstrate 5HT2C agonists' potential for management of atypical antipsychotic-related weight gain.
\end{abstract}

Keywords: Lorcaserin; Weight gain; Atypical antipsychotics; Olanzapine; Metabolic syndrome; Complications

\section{Introduction}

Weight gain is a common side effect associated with antipsychotics, particularly atypical antipsychotics. This poses a number of challenges to successful treatment, including medical complications of metabolic syndrome as well as potential for decreased adherence due to this unwanted side effect. Weight loss is achieved by decreasing caloric intake, not increasing energy expended, therefore medications reducing food cravings and promoting feelings of satiety could be a valuable tool for helping patients with antipsychotic-induced weight gain.

Exactly how antipsychotics contribute to weight gain is not fully understood, but their antagonism of the $5 \mathrm{HT} 2 \mathrm{C}$ receptor has been linked to increased propensity for weight gain. The 5HT2C receptor modulates activity in the hypothalamic proopiomelanocortin neurons, which influence perception of satiety. 5HT2C agonism decreases food cravings and increases feelings of fullness. Mice models missing these receptors were more likely to be obese, whereas mice models with increased expression of these receptors had decreased food intake and lower body weights compared to controls. 5HT2C modulation appears to be an important modulator of obesity particularly in high-fat diets as opposed to leptin-based modulation, which plays a more prominent role in obesity when subjects follow a carbohydrate-rich diet [1-5].

Lorcaserin is a selective 5HT2C agonist, FDA-approved for adult weight management in conjunction with low-calorie diet and exercise regimen. It was hypothesized in the following two case studies that lorcaserin's agonist activity may counteract the 5HT2C antagonism of olanzapine, helping to reduce appetite, and aid weight loss in patients that have experienced significant weight gain as a result of this psychotropic medication.

\section{Case Presentation}

\section{Case 1}

A 29 year old Asian male with schizophrenia on risperidone longacting injectable $50 \mathrm{mg}$ every 2 weeks and divalproex $1500 \mathrm{mg}$ at bedtime started olanzapine $10 \mathrm{mg}$ at bedtime for persistent psychotic symptoms. Six months after starting olanzapine, patient had gained 62 pounds, with total weight 272 pounds (BMI 41.1). Lorcaserin 10mg bid was added and the patient was educated to follow a low carbohydrate diet. The patient didn't report any adverse events. Three months after starting lorcaserin $10 \mathrm{mg}$ bid, patient weighed 245.8 pounds, representing $9.6 \%$ weight loss (26.2 pounds). Patient's food cravings, as measured by the Food Cravings Inventory (FCI) decreased $50.5 \%$ compared to baseline. His fasting insulin level was 61.6 (baseline fasting insulin level wasn't obtained) (Table 1).

\section{Case 2}

A 35 year old Hispanic male with schizophrenia on risperidone 4 $\mathrm{mg}$ at bedtime started olanzapine $10 \mathrm{mg}$ at bedtime for uncontrolled psychotic symptoms. Four months later, patient gained 26 pounds, with total weight 221 pounds (BMI 31.7). One week after starting lorcaserin $10 \mathrm{mg}$ bid, patient lost 5 pounds and reported $21 \%$ reduction in food cravings by FCI. Patient was unable to maintain low-carbohydrate diet; his weight increased to 225.6 pounds twelve weeks after he started treatment $(2.1 \%$ increase compared to weight prior to starting lorcaserin). Lorcaserin was subsequently discontinued due to lack of response in accordance with the label. Six weeks after discontinuing lorcaserin, patient weighed 234.6 pounds (4\% gain from weight at discontinuation) as well as $28 \%$ increase in food craving. As a result, the olanzapine was discontinued at that time (Table 2).

\begin{tabular}{|c|c|c|c|}
\hline & Initial & 3 months later & 14 months later \\
\hline Chol & 182 & 133 & 151 \\
\hline HDL & 37 & 27 & 35 \\
\hline Triglyceride & 434 & 223 & 138 \\
\hline LDL & 93 & 61 & 88 \\
\hline HbA1C & 5.7 & 4.6 & 5.3 \\
\hline Insulin & None & 61.6 & 6.5 \\
\hline AST & 47 & None & 18 \\
\hline ALT & 46 & None & 20 \\
\hline Weight & 272 & 245.8 & 225.8 \\
\hline
\end{tabular}

Table 1: Case 1.

*Corresponding author: Kevin Rimal Bera, University of Southern California 41 Exploration Irvine, CA 92618, USA, Tel: +1-949-933-0380; E-mail: Kevinbera24@gmail.com

Received October 26, 2015; Accepted November 14, 2015; Published November 16,2015

Citation: Nguyen C, Suzuki A, Bera K (2015) Use of Lorcaserin, A 5HT2C Agonist, In the Management of Olanzapine-Induced Weight Gain. J Metabolic Synd 4: 190 doi:10.4172/2167-0943.1000190

Copyright: $\odot 2015$ Nguyen C, et al. This is an open-access article distributed under the terms of the Creative Commons Attribution License, which permits unrestricted use, distribution, and reproduction in any medium, provided the original author and source are credited. 


\begin{tabular}{|c|c|c|c|}
\hline & Initial & 3 months later & 12 months later \\
\hline Chol & 215 & None & 176 \\
\hline HDL & 53 & None & 43 \\
\hline Triglyceride & 180 & None & 194 \\
\hline LDL & 126 & None & 94 \\
\hline HbA1C & 5.7 & None & 5.8 \\
\hline AST & None & None & 32 \\
\hline ALT & None & None & 39 \\
\hline Weight & 221 & 225.6 & 229.4 \\
\hline
\end{tabular}

\section{Treatment}

Both patients were initiated on lorcaserin $10 \mathrm{mg}$ twice a day and instructed to follow a low carbohydrate diet.

\section{Outcome and Follow-Up}

Case 1: The patient continued treatment with lorcaserin for a total of 14 months. He lost another 20 pounds, weighing at 225.8 pounds. His AST and ALT improved as well as his cholesterol, triglyercides, HDL, and LDL. His fasting insulin normalized at 6.5.

Case 2: After stopping the olanzapine, the patient reported feeling less hungry. He didn't gain any more weight and was able to lose about 5 pounds. Twelve months after he started lorcaserin, the patient weighed 229.4 pounds. His cholesterol, triglyecerides, HDL and LDL improved.

\section{Discussion}

While lorcaserin is FDA-approved for chronic weight management in adults, it has not been studied specifically in populations dealing with antipsychotic-induced weight gain.

The patient in Case 1 demonstrated a substantial decrease in food cravings as well as clinically significant weight loss $(9.6 \%$ weight loss). The patient attributed the decrease in food cravings to helping him follow the low carbohydrate diet. The patient had significant reduction in his fasting insulin level. The mechanism of improvement is unknown, but we postulate that a change in diet may be a huge contributing factor.

The patient in Case 2 initially showed a similar response; however, he ultimately was unable to follow the low-carbohydrate diet and continued to gain weight. The patient didn't have stable housing so he was unable to cook his own meals. He ate at soup kitchens or fast foods that contained starchy carbohydrates. Even though the patient didn't have as much food cravings, his limited food choices led him to eat high caloric foods. In addition, the patient admitted to medications noncompliance due to his unstable living situation. This may have contributed to an increase in food cravings, leading to weight gain.

It is noteworthy, however, that patient's had gained more weight after discontinuing lorcaserin than he did while taking this medication ( $4 \%$ increase after discontinuing medication versus $2.1 \%$ increase while taking lorcaserin). He also reported increased food cravings while off the medication while he was still taking olanzapine. After olanzapine was discontinued; the patient reported fewer cravings and was able to lose some weight on his own.

There were multiple factors contributing to patient's continued weight gain in case 2. Patient's inability to adhere to the recommended diet was likely a major contributing factor, but it is important to note that olanzapine acts on receptors other than 5HT2C that are also associated with appetite and weight. Olanzapine has strong antagonist activity at the histamine- 1 receptor, which has been correlated to weight gain. Olanzapine has antagonistic activity at peripheral M3 muscarinic receptor, which has been shown to have a dose-dependent connection to obesity [6]. Further exploration of these mechanisms may help identify which patients will benefit most from interventions like lorcaserin.

The results in these two cases suggest that Lorcaserin's selective 5HT2C agonist properties can counteract olanzapine's 5HT2C antagonism, resulting in increased satiety. This has implications for facilitating weight loss or at the least, mitigating weight gain while taking olanzapine.

\section{Conclusion}

- Lorcaserin has potential as an effective treatment to reduce food cravings and facilitate weight loss in patients experiencing olanzapineinduced weight gain.

- Although lorcaserin yielded promising initial results in case 2, patient's inability to adhere to diet suggests that other mechanisms contributing to weight gain must continue to be explored.

- The proposed mechanism by which lorcaserin facilitates weight loss is that its agonist effect counteracts olanzapine's antagonistic effect on $5 \mathrm{HT} 2 \mathrm{C}$ receptors.

\section{References}

1. Praharaj SK, Jana AM, Goyal N (2011) Metformin for olanzapine-induced weight gain: a systematic review and meta-analysis. $\mathrm{Br} \mathrm{J}$ Clin Pharmacol 70 377-382.

2. Wu R, Zhao J, Jin H (2008) Lifestyle Intervention and Metformin for treatment of anyipsychotic induced weight gain, A randomized controlled trial. JAMA 299: 185-193.

3. Alvarez-Jimenez M, Gonzalez-Blanch C, Vazquez-Barquero JL, Pérez-Iglesias R, Martínez-García O, et al. (2006) Attenuation of antipsychotic induced weight gain with early behavroral intervention in drug-naïve first-episode psychosis patients: a randomized controlled trial. J Clin Psychiatry 67: 1253-1260.

4. Allison DB, Mentore JL, Heo M, Chandler LP, Cappelleri JC, et al. (1999) Antipsychotic-induced weight gain: a comprehensive research synthesis. Am J Psychiatry 156: 1686-1696.

5. Kirk S, Glazebrook J, Grayson B (2009) Olanzapine-induced weight gain in the rat: role of $5-\mathrm{HT} 2 \mathrm{C}$ and histamine $\mathrm{H} 1$ receptors. Psychopharmacology 207: 119-125.

6. Smith SR, Weissman NJ, Anderson CM, Sanchez M, Chuang E, et al. (2010) Multicenter, placebo-controlled trial of lorcaserin for weight management. $\mathrm{N}$ Eng J Med 363: 245-256. 